\title{
Volume fraction instability in an oscillating non-Brownian iso-dense suspen- sion
}

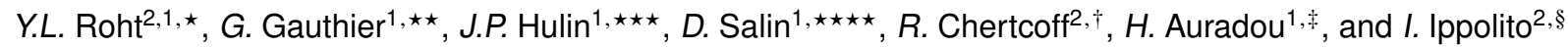 \\ ${ }^{1}$ Laboratoire FAST, Univ. Paris Sud, CNRS, Université Paris-Saclay, F-91405, Orsay, France \\ ${ }^{2}$ Universidad de Buenos-Aires, Facultad de Ingeniería, Grupo de Medios Porosos, CONICET, Paseo Colón 850, 1063, Buenos \\ Aires (Argentina).
}

\begin{abstract}
The instability of an iso-dense non-Brownian suspension of polystyrene beads of diameter $40 \mu \mathrm{m}$ dispersed in a water-glycerol mixture submitted to a periodic square wave oscillating flow in a Hele-Shaw cell is studied experimentally. The instability gives rise to stationary bead concentration waves transverse to the flow. It has been observed for average particle volume fractions between 0.25 and 0.4 , for periods of the square wave flow variation between 0.4 and $10 \mathrm{~s}$ and in finite intervals of the amplitude of the fluid displacement. The study shows that the wavelength $\lambda$ increases roughly linearly with the amplitude of the oscillatory flow; on the other hand, $\lambda$ is independent of the particle concentration and of the period of oscillation of the flow although the minimum threshold amplitude for observing the instability increases with the period.
\end{abstract}

\section{Introduction}

Dense suspension flows are encountered in various industrial processes (food processing, nuclear waste reprocessing, manufacturing of concrete or reinforced plastics ...), in biological (blood tests) or environmental engineering (wastewater treatment). This large area of applications gave rise to numerous studies both theoretical, numerical or experimental. However, some properties of suspension flows are not yet fully understood such as shear induced migration [1] and more precisely the dynamics of this migration in axisymmetric Poiseuille flows at moderate volume fractions $(\phi \leq 0.3)$ [2]. Moreover recent numerical simulations [3] point out the strong influence of confinement on the suspension rheology. On the other hand, several studies of dry granular media demonstrate that vibration may induce volume fraction instabilities [4] and the build-up of patterns has already been observed in oscillating suspensions, both experimentally $[5,6]$ and in numerical simulations [7]. These latter studies dealt with dilute suspensions (volume fraction $\phi \leq 10 \%$ ) of buoyant beads $(\Delta \rho \neq 0)$ oscillating in bulk at moderate frequencies $(f \geq 6 \mathrm{~Hz})$.

The present study deals with the different case of viscous iso-dense suspensions of moderate volume fraction $(\phi \geq 0.3)$ oscillating at low frequencies $(f \leq 2 \mathrm{~Hz})$ : these

\footnotetext{
^e-mail: lucreroht@gmail.com

$\star \star$ e-mail: georges.gauthier@u-psud.fr

$\star \star \star$ e-mail: hulin@ fast.u-psud.fr

$\star \star \star \star$ e-mail: salin@fast.u-psud.fr

†e-mail: ricardochertcoff@gmail.com

†e-mail: auradou@fast.u-psud.fr

$\S_{\text {e-mail: iippoli@fi.uba.ar }}$
}

suspensions are confined inside a Hele-Shaw cell of aperture $H=0.4 \mathrm{~mm}$ while the bead diameter is equal to $40 \mu \mathrm{m}$. The study is focused on the instability which develops in a finite range of periods and amplitudes and is marked by the appearance of periodic variations of the volume fraction $\phi$ of the suspension in the direction of the flow while it remains constant in the perpendicular direction. We shall study particularly the dependence of the wavelength of these structures on the period and amplitude of the flow, as well as on the concentration of the suspension.

\section{Experimental set-up and data processing}

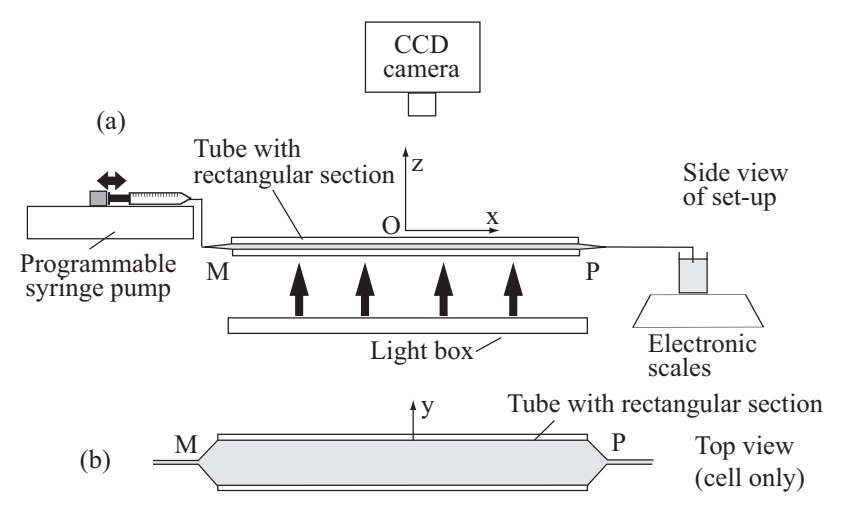

Figure 1. Schematic view of the experimental set-up

The experimental set-up is shown in Fig. 1. The suspension is contained in a Hele-Shaw cell of length 
$L=100 \mathrm{~mm}$, thickness $H=0.4 \mathrm{~mm}$ and aspect ratio $W / H=20$. The suspension is made of polystyrene beads of diameter $2 a=40 \mu \mathrm{m}$ immersed in a water glycerol mixture (mass concentration of glycerol $c=21 \%$ ) of viscosity $\eta=1.8 \mathrm{mPa} \cdot \mathrm{s}$ and with a density $\rho=1050 \mathrm{~kg} \cdot \mathrm{m}^{-3}$ matching that of the particles at a temperature of $23^{\circ} \mathrm{C}$. The oscillating flow of the suspension is induced by a programmable syringe pump fitted with $2.5 \mathrm{ml}$ glass syringes: the variation of the flow rate with time is a symmetrical square wave of period $T \geq 0.4 \mathrm{~s}$ adjustable by steps of $0.1 \mathrm{~s}$. The peak-to-peak amplitude $A$ of the mean displacement of the fluid in the cell has been varied between $A=0.2 \mathrm{~mm}$ and $A=5 \mathrm{~mm}$. The cell is horizontal (with its thickness parallel to the direction $z$ of gravity) and illuminated from below. Images of the patterns induced by the instability are acquired by a Nikon D300s camera located at $10 \mathrm{~cm}$ above the cell and used in the movie mode: it captures 25 frames per second with a resolution of $1920 \times 1080$ pixels.

Fig. 2a displays a typical view from above of a part of the cell at a time such that a stationary regime of the instability generated by the oscillations of the flow along $x$ has been reached: the instability leads to the appearance of periodic bands transverse to the flow. These bands are detected from variations of the transmitted light intensity which indicates that they correspond to variations of the particle concentration. The image has been enhanced by applying a low pass filter (smoothing out variations of the optical density in the $x$ direction due to the bands) and by subtracting the filtered image from the original one: this eliminates low frequency variations and enhances the contrast of the bands.

As one can see on Fig. 2a, the bands are more visible and straighter in the region of the axis of symmetry: in order to reduce the influence of the noise of the image, we average therefore, for a given value of $x$, the grey levels of all pixels located within a range of distances $y$ of width $\delta y \sim 0.1 \mathrm{~W}$ close to the axis. The averages $I(x, t)$ obtained for the different distances $x$ and times $t$ are plotted as grey levels in the spatiotemporal diagram of Fig. 2b. The zigzag shape of the bands visible in the diagram reflects the periodic displacement along $x$ of the volume fraction variations induced by the periodic flow: the peak-to-peak amplitude $A$ of the displacements of the bands is used to characterize the displacement of the fluid imposed by the oscillating flow.

Finally, we compute, for all times $t$, the autocorrelation function:

$$
F(\delta x, t)=\int I(x, t) \times I(x-\delta x, t) \mathrm{d} x .
$$

The function $F(\delta x, t)$ is plotted as grey levels in the spatiotemporal diagram of Fig. 2c as a function of time and of the interval $\delta x . F(\delta x, t)$ is symmetrical with respect to $\delta x=0$ so that only the right part of the diagram has been plotted. The diagram displays alternate light and dark vertical bands: their period corresponds to the wavelength $\lambda$ of the variations of the volume fraction induced by the instability. The visibility of the bands decreases with $\delta x$
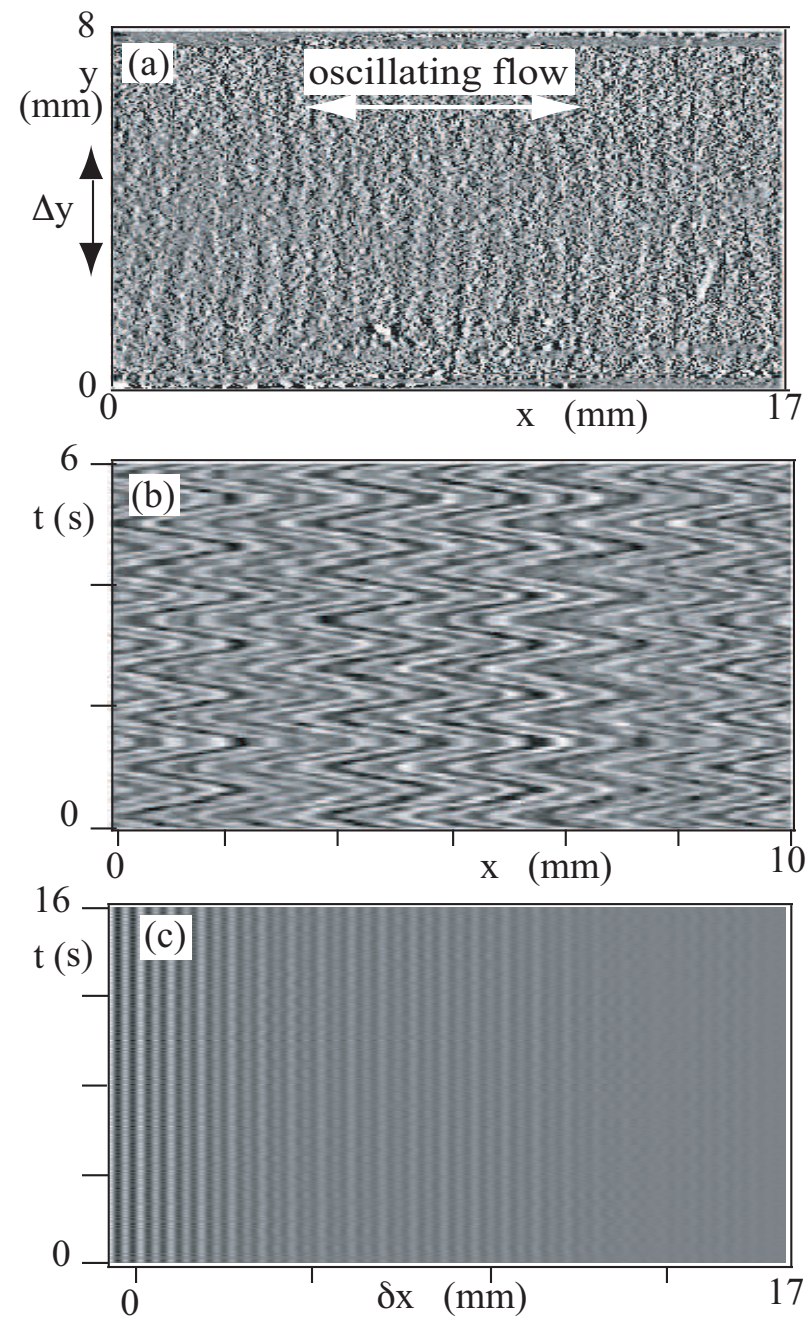

Figure 2. a) View from above of the instantaneous transmitted light pattern produced by the volume fraction instability of a suspension of polystyrene beads $(\phi=0.35)$ submitted to an oscillatory flow ( $T=0.8 \mathrm{~s}, A=2.34 \mathrm{~mm}$ ) parallel to the direction $x$. Grey level variations correspond to those of the volume fraction. b) Spatiotemporal diagram of the local transmitted light intensities averaged over an interval $\Delta y=0.1 \mathrm{~W}$ in the transverse direction (grey level); vertical scale: time; horizontal scale: coordinate $x$ along the flow direction. c) Spatiotemporal diagram of the autocorrelation function (grey levels) of the profiles of Fig. b; vertical scale: time; horizontal scale: interval $\delta x$ (Eq. 1).

over a distance which corresponds to the spatial correlation length of the instability: depending on the experiment it may range from $5 \lambda$ to $30 \lambda$. Practically, $\lambda$ is determined by plotting the distances $\delta x$ corresponding to the different maxima of $F$ as a function of their number and performing a linear regression. In some of the diagrams of the type of Fig. $2 c$ there is a small undulation of the vertical bands at large values of $\delta x$ : this corresponds to a weak modulation of the wavelength by the oscillating flow.

\section{Experimental results}

Periodic bands transverse to the flow marking the occurrence of the instability were observed only for suspen- 


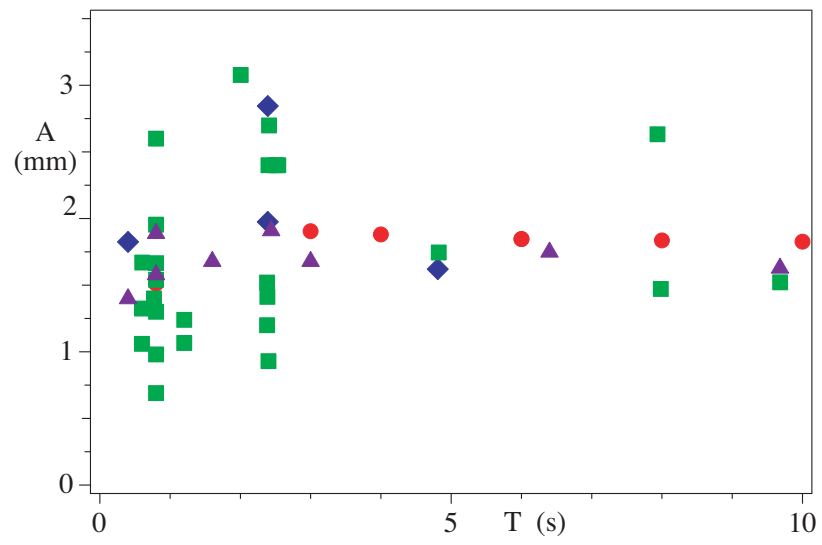

Figure 3. Map of the pairs of values of the amplitude $A$ of the instability pattern oscillations and of the period $T$ for which the instability has been observed in this study. Flowing fluid: $40 \mu \mathrm{m}$ polystyrene particles in a $21 \%$ water-glycerold solution; particle fractions: $\phi=0.25(\diamond), \phi=0.3(\bullet), \phi=0.35(\square), \phi=0.37(\Delta)$. Cell dimensions: $W=8 \mathrm{~mm}, H=0.4 \mathrm{~mm}, L=100 \mathrm{~mm}$.

sions of large enough volume fraction $\phi \geq 0.25$ and up to $\phi=0.37$. This latter upper limit of $\phi$ may actually reflect other effects such as the blockage of the suspension in the cell, at the outlet of the syringe or in the connecting tubes. Moreover, the intensity of the transmitted light decreases with the concentration which makes the patterns less visible and may account in part for their apparent disappearance.

We have studied the domain of existence of the instability which is displayed on Fig. 3. For an average volume fraction $\phi=0.35$ ((ष) symbol on Fig 3) and for the shortest imposed period $T=0.4 \mathrm{~s}$, the instability appears above a threshold amplitude $A_{c}=0.8 \mathrm{~mm}$. The instability pattern remains visible when $A$ increases up to an upper limit $A_{l} \simeq 3 \mathrm{~mm}$ above which it disappears and the suspension is no longer organized. Moreover, the lower threshold $A_{c}$ increases slowly with the period $T$ and reaches a plateau value $A_{c} \simeq 1.4 \mathrm{~mm}$ for $T \gtrsim 5 \mathrm{~s}$. For lower volume fractions $\phi$, the minimum amplitude $A$ required for observing the instability increases: for $\phi=0.25$ and $T=0.4 \mathrm{~s}$, for instance, $A_{c}=1.3 \mathrm{~mm}$.

As mentioned above, the wavelength $\lambda$ of the sequence of bands at a given time is determined from the interval between the values of $x$ corresponding to the maxima of the autocorrelation function $F(\delta x, t)$ defined by Eq. 1. A stationary flow and volume fraction variation regime may be assumed to be reached after $\sim 10-20$ oscillations and $\lambda$ remains constant thereafter within some cases, a small modulation by $1-2 \%$ of same period as that of the flow.

Figures $4 \mathrm{a}-\mathrm{b}$ display respectively for several average concentrations $(0.25 \leq \phi \leq 0.37)$ the variation of the wavelength $\lambda$ as a function of either the period $T$ or the measured amplitude $A$.

The amplitude $A$ is approximately the same $(A=$ $1.75 \pm 0.25 \mathrm{~mm}$ ) for all data points of Graph 4a: we observe that $\lambda$ is nearly constant with the period $T$ for all three particle fractions ( $\phi=0.3,0,35$ and 0.37$)$. More-
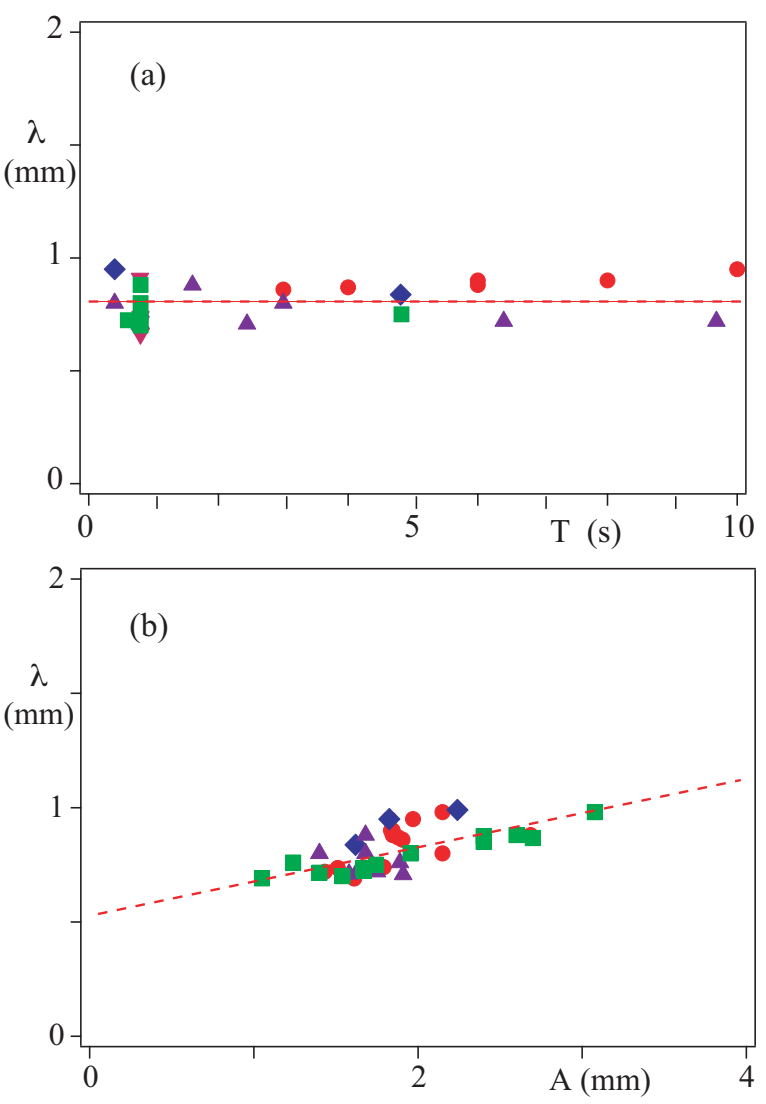

Figure 4. Variation of the average wavelength $\lambda(\mathrm{mm})$ of the instability as a function of: (a) the period $T$ of the flow $(A=$ $1.75 \pm 0.25 \mathrm{~mm}$ ) and: (b) the peak-to-peak amplitude $A$ of the displacement of the instability pattern $(0.4 \leq T \leq 10 \mathrm{~s})$. The flowing solutions, the cell and the meaning of the symbols are the same as in Fig. 3.

over, there does not seem to be any systematic variation of $\lambda$ with $\phi$.

Since Graph 4a indicates that $\lambda$ is independent of $T$, the data points corresponding to all values of $T$ have been superimposed in Graph $4 \mathrm{~b}$ as a function of $A$ : the good collapse of the data points confirms this lack of dependence of $\lambda$ on $T$. This graph shows that $\lambda$ increases instead roughly linearly by about $25 \%$ when $A$ increases from 1 to $3 \mathrm{~mm}$ with: $\lambda \simeq 0.54+0.14 \times A \mathrm{~mm}$. It also confirms that there is no clear variation of $\lambda$ with the particle volume fraction $\phi$ for a given amplitude $A$.

\section{Discussion and conclusion}

We have identified and analyzed an instability of iso-dense non Brownian suspensions in a Hele-Shaw cell submitted to an oscillatory flow velocity with a square wave variation at a low frequency. This instability occurs only for large enough mean volume fractions of the particles (phi $\geq 0.25$ ). On the one hand, the wavelength $\lambda$ of this instability is independent of the period of the oscillations and of the particle volume fraction; on the other hand, the minimum threshold amplitude for observing the bands in- 
creases with the period $T$ while it decreases at high volume fractions.

This instability of an oscillating suspension differs from those reported by other authors in one or several of its characteristics: lower frequency, larger volume fraction, $1 D$ instability pattern with straight parallel bands. The periodic patterns studied in the present work are also different in nature from those resulting from an oscillating fluid shear flow above a granular sediment $[8,9]$ : such sediments might indeed appear due to sedimentation induced by a small residual density difference of the beads and the fluid or to the suspension getting structured with a sediment layer close to the wall and clear fluid between them $[10,11]$.

In order to eliminate the possible effect of gravity, several experiments have been performed, holding the cell with the $y$-axis vertical (Fig. 1: band patterns transverse to the horizontal flow and with the same wavelength as for horizontal cells were observed like in the latter.

Also, realizing experiments with a non-zero density contrast between the beads and the fluid hinders the appearance of the instability rather than fostering it. For a relative density contrast $\Delta \rho / \rho=1.5 \%, A_{c}$ is significantly increased; for larger $\Delta \rho / \rho$ values, the periodic pattern does not appear any more.

Finally, a few preliminary visualizations of the distribution of the particles in the gap of the cell have been performed by means of a fluorescent dye dissolved in the fluid and of a laser sheet perpendicular to the cell: the small variations of the volume fraction $\phi(x, t)$ due to the instability resulted in variations of the intensity of the fluorescence light. These variations spanned the whole thickness of the cell and no layering effect was observed. The instability appears therefore as a succession of particles plugs that might result from shear induced migration: this implies that the important control parameter of the process would be the ratio $A / H$ [12]. In order to test this hypothesis, further experiments will have to be performed with cells of different thicknesses. More information on the parameters determining the domain of existence of the instability and its wavelength is also needed: it will be obtained by varying the size and density of the particles as well as the density and viscosity of the fluid and the geometry of the flow cell (thickness, length and width). A more lo- cal analysis of the distribution of the particles will also be necessary to understand the processes involved.

\section{Acknowledgements}

We acknowledge support by the RTRA Triangle de la Physique, by the LIA PMF-FMF (FrancoArgentinian International Associated Laboratory in the Physics and Mechanics of Fluids) and from UBACyT 20020130100570BA. The thesis of Y.L. Roht is supported by a fellowship from the Peruilh foundation of the Faculty of Engineering of the Buenos-Aires University, by a Bec.Ar fellowship and by an Eiffel fellowship. The authors want to thank A. Aubertin, L. Auffray and R. Pidoux for their help in the design and realization of the experimental set-up and J.E. Wesfreid for helpful discussions.

\section{References}

[1] D. Leighton, A. Acrivos, Journal of Fluid Mechanics 181, 415 (1987)

[2] B. Snook, J.E. Butler, É. Guazzelli, Journal of Fluid Mechanics 786, 128 (2016)

[3] S. Gallier, E. Lemaire, F. Peters, L. Lobry, Phys. Rev. E 92, 020301 (2015)

[4] D. Krengel, S. Strobl, A. Sack, M. Heckel, T. Pöschel, Granular Matter 15, 377 (2013)

[5] P. Gondret, L. Petit, Physics of Fluids 8, 2284 (1996)

[6] R. Moosavi, M. Maleki, M.R. Shaebani, J.C. RuizSuárez, E. Clément, EPL (Europhysics Letters) 107, 34006 (2014)

[7] V. Loisel, M. Abbas, O. Masbernat, E. Climent, Physics of Fluids 27, 123304 (2015)

[8] G. Rousseaux, J. Kruithof, P. Jenffer, J.E. Wesfreid, Physical Review E 78, 016302 (2008)

[9] M. Abedi, M.A. Jalali, M. Maleki, Journal of Fluid Mechanics 758, 312 (2014)

[10] J.E. Butler, P.D. Majors, R.T. Bonnecaze, Physics of Fluids 11, 2865 (1999)

[11] J.F. Morris, Physics of Fluids 13, 2457 (2001)

[12] P. Nott and J. Brady, Journal of Fluid Mechanics 275, 157 (1994) 